\title{
Unanswered questions regarding the management of sunitinib-induced hypothyroidism
}

\author{
David Garfield ${ }^{\star}$, Aleck Hercbergs and Paul Davis
}

$D$ Garfield is a medical oncologist affiliated with the University of Colorado Health Sciences Center, A Hercbergs is a Radiation Oncologist at the Cleveland Clinic Foundation, Cleveland, $\mathrm{OH}$, and $P$ Davis is Senior Associate Dean for Clinical Research and Professor of Medicine at Albany Medical College, Albany, NY, USA.

\section{Correspondence \\ *Department of Medicine University of Colorado Health Sciences Center \\ 57 Hyde Park Circle \\ Denver \\ CO 80209 \\ USA \\ david.garfield@earthlink.net}

Received 20 June 2007

Accepted 17 September 2007 Published online

23 October 2007

www.nature.com/clinicalpractice doi:10.1038/ncponc0998
Hypothyroidism seems to be a risk of sunitinib therapy for cancer. We review the evidence for the occurrence of hypothyroidism in this setting and discuss possible actions of thyroid hormone replacement in patients with cancer.

Multitargeted tyrosine kinase inhibitors are novel oral chemotherapeutic agents with efficacy in treating advanced renal cell carcinoma (RCC) and gastrointestinal stromal tumors, among other cancers. Prolonged administration of some tyrosine kinase inhibitors, such as sunitinib, however, might lead to decreased thyroid function. The common adverse event of fatigue in sunitinib-treated patients reflects drug-induced hypothyroidism in $30-40 \%$ of individuals who experience such symptoms. ${ }^{1,2}$ Sunitinib inhibits thyroid peroxidase in a similar fashion to propylthiouracil (PTU), albeit to only one-quarter the degree. ${ }^{3}$ In preclinical models, administration of various VEGF pathway inhibitors caused marked thyroid capillary fenestration and regression, with an associated rise in thyroid-stimulating hormone (TSH); ${ }^{4}$ therefore, patients with RCC and pre-existing thyroid disease might be particularly susceptible to hypothyroidism while receiving sunitinib. The normal thyroid gland contains several months' store of thyroid hormone and would explain the delay in onset of hypothyroidism seen in patients receiving sunitinib. ${ }^{1,2}$ Imatinib use in thyroidectomized cancer patients with hypothyroidism might also increase the dose of replacement thyroid hormone needed, ${ }^{5}$ suggesting that imatinib affects disposal of thyroid hormone. What is apparent is that hypothyroidism can complicate the management of solid tumors with tyrosine kinase inhibitors, and thyroid hormone replacement has been, therefore, routinely recommended in this setting. ${ }^{1,2}$

The use of any thyroid hormone therapy could be problematic in that there is epidemiologic and clinical evidence to suggest that the hypothyroid state may, in fact, confer benefit in the management of various carcinomas. In breast cancer, hypothyroidism has been associated with a reduced incidence of the cancer, older age at diagnosis, and disease that seems less aggressive. ${ }^{6}$
In patients with recurrent primary brain tumors, intentional induction of mild hypothyroidism has been associated with tumor regression and statistically significant prolongation of survival. ${ }^{6}$ There is also an association between the development of hypothyroidism and improved survival in patients with head and neck cancer. ${ }^{7}$ In general, patients with metastatic RCC and a history of hypothyroidism seem to do better with cancer treatment than those without such a history. ${ }^{6}$

Hypothyroidism has been noted in patients with RCC treated with interleukin (IL-) 2 or interferon alfa, and with IL-2 therapy there was an association between development of hypothyroidism and tumor response. ${ }^{8}$ Bexarotene, a synthetic retinoid that may be useful in the treatment of non-small-cell lung cancer, has been associated with hypothyroidism. ${ }^{9}$ In the most recent study of bexarotene, thyroid hormone was given prophylactically. ${ }^{10}$ On the basis of the evidence cited above and in vitro descriptions of the effect of thyroid hormone on tumor cell proliferation, we suggest that such administration of thyroid hormone should not be routine. Rather, the clinical evidence we have cited indicates that thyroid hormone may be a 'permissive' factor in tumor growth in patients with active or previously treated cancer in remission. Preclinical studies in various transplantable solid tumor types have shown that growth and proliferation rates accelerate in response to thyroid hormone. ${ }^{8}$

A cell membrane receptor for thyroid hormone, $\alpha_{\mathrm{V}} \beta_{3}$ integrin, mediates the effect of physiological concentrations of the hormone in vitro on human and murine tumor cell proliferation. ${ }^{11}$ This integrin receptor is also required for the induction of angiogenesis by the thyroid hormone, which may be tumor-relevant. ${ }^{6}$ The proangiogenic effect of this hormone, initiated at the cell surface, involves induction of basic fibroblast growth factor release by endothelial cells ${ }^{6}$ and VEGF release from endothelial, fibroblastic, tumor and other cells.

MAPK1/2 signaling results in angiogenesis and tumor cell proliferation. Inhibition of thyroidhormone-binding at the integrin receptor by 
tetraiodothyroacetic acid (tetrac) prevents activation of MAPK1/2 by thyroid hormone and the downstream consequences of MAPK1/2 activation, including hormonal induction of angiogenesis and tumor cell proliferation. Tetrac is a derivative of L-thyroxine that has only inhibitory effects at the integrin receptor. ${ }^{11}$ However, the nuclear receptor for thyroid hormone is not primarily involved in these effects of the hormone. It should be noted that most hypothyroid patients receiving thyroid hormone replacement will experience an increase in circulating levels of VEGF. ${ }^{12}$

Other possible permissive mechanisms of action of thyroid hormone on cancer cells include amplification of epidermal growth factor activity at its receptor, ${ }^{6}$ stimulation of tumor cell migration, and a direct trophic effect on tumor cells. In addition, thyroid hormone can directly prevent p53-dependent apoptosis, ${ }^{11}$ as well as prevent apoptosis through phosphorylation of insulin-like growth factor 1 receptor. ${ }^{6}$ Moreover, there are thyroid hormone receptors on mitochondria, and deficiency of thyroid hormone can lead to apoptosis. Abrogation of these antiapoptotic effects could make chemotherapy and radiation therapy more effective.

The VEGF pathway is upregulated in the majority of patients with RCC, and sunitinib is used to disrupt this pathway. Thus, the routine use of thyroid hormone as replacement therapy in patients with sunitinib-induced hypothyroidism could lead to an increase in VEGF, perhaps negating the salutary effects of the tyrosine kinase inhibitor and perhaps other anticancer agents. With an estimated 20 million individuals in the US presently receiving thyroid hormone, the hormone's concurrent use with other therapies in patients with any active or prior cancer should be considered carefully. Indeed, a clinical study using the inactive thyroid hormone agonist tetrac would be of some interest. ${ }^{11}$

We believe that the issue of any beneficial role of hypothyroidism and subsequent risk of giving thyroid hormone replacement therapy needs to be addressed and answered. This should be done before any suggestion is made that thyroid hormone should be given automatically to patients with solid tumors who are receiving either sunitinib or other kinase inhibitors in order to achieve a euthyroid state. Alternatively, a recommendation could be that, until such studies are completed, as little thyroid hormone is administered as possible. Monthly testing of TSH levels also seems reasonable.

The American Thyroid Association suggests that thyroid hormone should be withheld in asymptomatic, chemically-only hypothyroid patients (serum TSH concentration of $5-10 \mathrm{mIU} / \mathrm{l}) .{ }^{13} \mathrm{It}$ is noteworthy that some patients with serum TSH levels above $10 \mathrm{mIU} / 1$ have few or no symptoms of hypothyroidism and, therefore, do not necessarily need replacement therapy. On the other hand, fatigue in cancer patients is often multifactoral, so the decision to start thyroid hormone can be problematic. In such cases, a therapeutic trial of low-dose thyroid hormone replacement may be conducted. In the setting of most thyroid cancers, however, suppression of endogenous TSH secretion remains the treatment goal.

\section{References}

1 Rini Bl et al. (2007) Hypothyroidism in patients with metastatic renal cell carcinoma treated with sunitinib. J Nat/ Cancer Inst 99: 81-83

2 Desai J et al. (2006) Hypothyroidism after sunitinib treatment for patients with gastrointestinal stromal tumors. Ann Intern Med 145: 660-664

3 Wong E et al. (2007) Sunitinib induces hypothyroidism in advanced cancer patients and may inhibit thyroid peroxidase activity. Thyroid 17: 351-355

4 Kamba T et al. (2006) VEGF-dependent plasticity of fenestrated capillaries in the normal adult microvasculature. Am J Physiol Heart Circ Physiol 290: $\mathrm{H} 560-\mathrm{H} 576$

5 de Groot JWB et al. (2005) Imatinib induces hypothyroidism in patients receiving levothyroxine. Clin Pharmacol Ther 78: 433-438

6 Davis PJ et al. (2006) Cell surface receptor for thyroid hormone and tumor cell proliferation. Expert Rev Endocrin Metab 1: 753-761

7 Nelson M et al. (2006) Association between development of hypothyroidism and improved survival in patients with head and neck cancer. Arch Otolaryngol Head Neck Surg 132: 1041-1046

8 Hercbergs A (1999) Spontaneous remission of cancer - a thyroid hormone dependent phenomenon? Anticancer Res 19: 4839-4844

9 Golden WM et al. (2007) Single-dose rexinoid rapidly and specifically suppresses serum thyrotropin in normal subjects. J Clin Endocrinol Metab 92: 124-130

10 Govindan R et al. (2006) Phase II trial of bexarotene capsules in patients with advanced non-small cell lung cancer after failure of two or more previous therapies. $J$ Clin Oncol 24: 4848-4854

11 Lin HY et al. (2007) Thyroid hormone is a MAPKdependent growth factor thyroid cancer cells and is anti-apoptotic. Steroids 72: 180-187

12 Dedecjus $M$ et al. (2007) Influence of L-thyroxine administration on poor-platelet plasma VEGF concentrations in patients with induced short-term hypothyroidism, monitored for thyroid carcinoma. Endocr J 54: 63-69

13 Surks ML et al. (2004) Subclinical thyroid disease: scientific review and guidelines for diagnosis and management. JAMA 291: 228-238
Competing interests

D Garfield declared associations with the following companies: Array BioPharma, AstraZeneca and Pharmatech. See the article online for full details of the relationships. The other authors declared no competing interests. 